\title{
Mobile health technologies and medical records
}

\author{
How the mobile phone is transforming healthcare in low-resource settings
}

Sean Wong, Wendy Wang

\begin{abstract}
Migrants and refugees often face healthcare difficulties that may be difficult to address in low-resource healthcare settings. They often present with multifaceted and complex health problems that pose unique challenges for both primary care providers and public health officials. Mobile health technologies (mHealth), which involves the use of mobile technology in order to support medical and public health practices, is currently being explored as a means of addressing some of these challenges. The rapid expansion of mobile technology and increasing ubiquity of devices such as mobile phones even in low-resource settings has made mHealth an attractive option for the provision and support of healthcare. mHealth has been implemented in novel ways to enhance patient education, support immunization, and monitor infectious disease in vulnerable populations. Despite its benefits, there remain limitations to the use of mHealth in lowresource healthcare settings, including concerns regarding personal health information security, user adherence, and validated implementation, amongst others. This article will explore the use of mHealth to support medical care in lowresource healthcare settings.
\end{abstract}

\section{INTRODUCTION}

The number of migrants and refugees in developed countries such as Canada has increased dramatically in recent years. The UN Commissioner for Refugees estimates that 59.5 million people globally have been forced from their home state as a direct result of conflict, violence, or human rights violations. ${ }^{1}$ Canada, as the ninth-largest recipient of asylum seekers worldwide, has taken in 40,081 refugees from Syria alone. ${ }^{2}$ These displaced individuals face unique challenges arising from culture shock, poverty, marginalization, and discrimination which hinder their access to healthcare and lead to long-term ramifications on individual health. For example, refugees may suffer from mental health problems such as depression or post-traumatic stress disorder, and physical ailments related to overcrowding and communicable diseases. ${ }^{3}$ These health issues are common in vulnerable populations exposed to poorly resourced and underfunded healthcare settings. Mobile health technologies (mHealth) have been suggested as a potential solution to insufficient healthcare in low-resource settings for refugee and migrant populations. This article will focus on mobile health technologies with respect to disease surveillance and patient management.

\section{WHAT ARE MOBILE HEALTH TECHNOLOGIES?}

Mobile health technologies (mHealth) are defined as medical and public health practices supported by mobile devices such as mobile phones, patient monitoring devices, personal digital assistants, and other wireless devices. ${ }^{4}$ mHealth can be considered a subcategory of electronic health, which refers to the use of all electronic technologies in medicine, including computers. ${ }^{5}$ Historically, healthcare technology has been linked with the development of more powerful and sophisticated computing hardware. One of the earliest uses of technology in healthcare was the development of the electronic medical record (EMR) as a replacement for paper records. While some of the first EMRs were developed as early as the 1960s, improvements in processing and computing hardware have led to their widespread dissemination in healthcare systems around the world. ${ }^{6}$ The rapid expansion of technology in the mobile phone sector paired with the highly accessible nature of mobile phones in the modern era have made mHealth technologies an attractive solution to healthcare problems faced by refugees in low-resource settings.

The increasingly universal nature of mobile devices has made mHealth an attractive option in tackling healthcare issues in low-resources settings. One study in a Syrian Za'atari refugee camp showed that approximately $90 \%$ of Syrians possessed a working mobile phone and $60 \%$ could access the internet through their smartphones. ${ }^{7}$ From a public health standpoint, another attractive feature of mHealth is the ease through which healthcare interventions may be implemented on a large scale in a language and culturally sensitive manner. Healthcare applications seem to be an underutilized feature of smartphone use in the refugee population, and thus may be a target for intervention. One survey of Government-assisted refugees in Canada found that while $92 \%$ of participants had access to a smartphone, only $14 \%$ of them were utilizing healthcare apps. ${ }^{8}$ As a result, mHealth has immense potential in that it can take advantage of what limited resources this population has in order to deliver best care practices.

\section{MHEALTH AND IMMUNIZATION}

Healthcare tracking with mobile devices is a trend that has taken the developed world by storm and has driven innovation in the mHealth space. For example, one is capable of tracking blood sugars and calculating carbohydrate intake in diabetic patients using minimally invasive patches paired with smartphone apps. ${ }^{9}$ While a similar level of sophistication may not be deliverable to a population of displaced individuals, the same concepts and ideas 
can be extrapolated to address their unique healthcare needs. One such need is the establishment of vaccination records and the provision of patient education on vaccine-preventable disease.

Newcomers are often subjected to convoluted travel patterns and may reside in overcrowded, unhygienic temporary residences for varying periods of time, making them susceptible to vaccinepreventable infectious diseases. ${ }^{10}$ Newcomers may also be hesitant to undergo vaccination for a variety of cultural and/or social reasons. The perception that immunization may conflict with cultural beliefs has been demonstrated as a significant cultural barrier to vaccination. Specifically, newcomers may be hesitant to receive the HPV vaccination for fear of encouraging sexual promiscuity. ${ }^{11}$ mHealth may be used as a tool to disseminate educational materials that address culture specific concerns in order to encourage preventative healthcare measures. In addition, newcomers may have difficulty accessing healthcare services because of language barriers and inadequate understanding of provider healthcare coverage. ${ }^{12}$ Establishing and maintaining accurate vaccination records provides a method of risk reduction that can be easily administered using mHealth to combat these issues. Smartphone apps such as the CANImmunize app enable newcomers to manage their own immunization information. This ranges from vaccination tracking for individuals and their dependents, to updates for upcoming vaccinations, to educational resources in a number of different languages. ${ }^{8}$ Furthermore, early exposure and normalization of vaccination practices in newcomer youth and children can ensure maintenance of helpful public health practices. ${ }^{13}$ Simple educational tools delivered through a smartphone application format have the potential to bridge knowledge gaps and improve public health in this vulnerable population.

\section{MHEALTH AND INFECTIOUS DISEASE MONITORING}

Large groups of displaced individuals place a unique stress on healthcare systems. Addressing healthcare concerns in this context can be taxing on providers, especially in low-resource settings. Such congregations are at higher risk for disease outbreak, and patients may have distinctive needs that are not typically in high demand. For instance, refugees displaced by violence would require an abundance of resources such as emergent care and specialized mental health services. Whereas well-funded and adequately resourced systems have the luxury of real-time data access for disease surveillance and resource re-allocation, their overloaded counterparts in newcomer settlements may not. This discrepancy in resource access has been identified as a target for mHealth intervention.

Many low-resource areas, such as refugee camps or rural areas in the developing world, still utilize paper-based healthcare information systems. The use of paper-based systems to record and submit infectious disease-related health events leads to delayed response by public health officials. ${ }^{14}$ There have been a number of pilot projects exploring the development communitybased participatory disease surveillance strategies using mHealth applications. One such project is the Southern African Centre for Infectious Disease Surveillance (SACIDS) AfyaData project, which utilized a simple smartphone app for reporting human and animal disease to health officials. ${ }^{15}$ Communities using the app were more likely to report instances of disease outbreak than those still using traditional paper-based reporting practices, leading to higher response rate from public health. Similar data reporting and collection tools based on simple smartphone functionality have been used to monitor disease incidence and even prescription practices in large scale gatherings. ${ }^{14}$ Utilizing $\mathrm{mHealth}$ to coordinate large-scale responses to healthcare crises such as a large influx of refugees and newcomers could be a cost-effective and feasible solution in a poorly resourced location.

\section{DRAWBACKS AND LIMITATIONS}

While mHealth offers an enticingly simple, cost-effective, and highly scalable means of responding to the healthcare needs of displaced individuals, there are several limitations. The effectiveness of mHealth with respect to data collection and medical records depends on buy-in and user adherence. Specifically, when relying on patient-reported data, there may be issues of inaccurate data input, misinterpretation of information, and literacy difficulties. ${ }^{16}$ Thus, the health data retrieved through mHealth initiatives may be less reliable in clinical decision-making, as it is subject to bias and confounding factors. Although the use of smartphone technology is constantly growing, there is still a large percentage of individuals who do not have access. While studies have shown that up to $90 \%$ of refugees in Canada have access to a smartphone, the remaining $10 \%$ would be excluded from any mHealth initiatives. Additional concerns have been raised regarding the privacy and confidentiality of data received through mHealth initiatives. Another major issue slowing the implementation of mHealth into general practice is the massive diversity of mobile applications. There are thousands available and even more being developed. Discovering applications that provide a secure and validated service requires significant time and resources. While mHealth is undoubtedly growing in popularity, its efficacy has still yet to be explored on a long-term, sustainable basis due to its novel implementation. ${ }^{14}$

\section{CONCLUSION}

Displaced individuals, including refugees and newcomers, face a number of unique health challenges. mHealth has the potential to be a ground-breaking development with respect to offering solutions to these issues, specifically in the areas of data collection and public health intervention. Two major examples of its early implementations include documentation and education on immunization initiatives and providing a means of disease surveillance in low-resource areas. While mHealth has immense potential to address gaps in healthcare, it currently lacks a significant body of evidence to support its efficacy. In conclusion, mHealth is still a developing field that, while promising, must still be evaluated critically as a means of delivering improved healthcare to displaced patients. 


\section{REFERENCES}

1. Langlois EV, Haines A, Tomson G, et al. Refugees: towards better access to health-care services. Lancet. 2016 Jan;387(10016):319-21. https://doi.org/10.1016/S0140-6736(16)00101-X

2. Oda A, Tuck A, Agic B, et al. Health care needs and use of health care services among newly arrived Syrian refugees: a cross-sectional study. CMAJ Open. 2017 May;5(2):E354-8. https://doi.org/10.9778/ cmajo.20160170

3. Burnett A. Asylum seekers and refugees in Britain: the health of survivors of torture and organised violence. BMJ. 2001 Mar;322(7286):606-9. https://doi.org/10.1136/bmj.322.7286.606

4. WHO. mHealth: New horizons for health through mobile technologies [Internet]. World Health Organization; 2011 [cited 2018 Nov 20]. Available from: https://www.who.int/goe/publications/goe_mhealth web.pdf

5. Dicianno BE, Parmanto B, Fairman AD, et al. Perspectives on the evolution of mobile (mHealth) technologies and application to rehabilitation. Phys Ther. 2015 Mar;95(3):397-405. https://doi. org/10.2522/ptj.20130534

6. Stead WW. Electronic health records. Stud Health Technol Inform. 2010;153:119-43.

7. Maitland $\mathrm{C}, \mathrm{Xu}$ Y. A social informatics analysis of refugee mobile phone use: a case study of Za'atari Syrian refugee camp. SSRN Electronic Journal. 2015 Mar; https://doi.org/10.2139/ssrn.2588300

8. Paradis M, Atkinson KM, Hui C, et al. Immunization and technology among newcomers: a needs assessment survey for a vaccine-tracking app. Hum Vaccines Immunother. 2018 Jul;14(7):1660-4. https://doi.org $\not 10.1080 / 21645515.2018 .1445449$.

9. Hirsch IB. Home blood glucose monitoring in type 2 diabetes: broken health care system undermines study's impact. Diabetes Care. 2011 Feb;34(2):527-8. https://doi.org/10.2337/dc10-2238.

10. Wilson L, Rubens-Augustson T, Murphy M, et al. Barriers to immunization among newcomers: a systematic review. Vaccine. 2018 Feb;36(8):1055-62. https://doi.org/10.1016/j.vaccine.2018.01.025.

11. Holman DM, Benard V, Roland KB, et al. Barriers to human papillomavirus vaccination among US adolescents: a systematic review of the literature. JAMA Pediatr. 2014 Jan;168(1):76-82. https://doi. org/10.1001/jamapediatrics.2013.2752

12. Kowal SP, Jardine CG, Bubela TM. "If they tell me to get it, I'll get it. If they don't....”: immunization decision-making processes of immigrant mothers. Can J Public Heal. 2015 Apr;106(4):e230-5. https://doi. org/10.17269/cjph.106.4803

13. Chen L, Du X, Zhang L, et al. Effectiveness of a smartphone app on improving immunization of children in rural Sichuan Province, China: a cluster randomized controlled trial. BMC Public Health. 2016 Aug;16:909. http:s//doi.org/10.1186/s12889-016-3549-0

14. Kazi DS, Greenough PG, Madhok R, et al. Using mobile technology to optimize disease surveillance and healthcare delivery at mass gatherings: a case study from India's Kumbh Mela. J Public Heal (Oxf.). 2017 Sep;39(3):616-24. https://doi.org/10.1093/pubmed/fdw091

15. Karimuribo ED, Mutagahywa E, Sindato C, et al. A smartphone app (AfyaData) for innovative one health disease surveillance from community to national levels in Africa: intervention in disease surveillance. JMIR Public Heal Surveill. 2017 Dec;3(4):e94. https://doi. org/10.2196/publichealth.7373.

16. Aranda-Jan CB, Mohutsiwa-Dibe N, Loukanova S. Systematic review on what works, what does not work and why of implementation of mobile health (mHealth) projects in Africa. BMC Public Health. 2014
Feb;14:188. https://doi.org/10.1186/1471-2458-14-188. 